\title{
Coronary aneurysms, myocardial dysfunction, and shock in a COVID-19 child, role of ECMO, immunomodulation, and cardiac CT
}

\section{Brief Report}

Cite this article: Mustafa MR, Carter MJ, Wong J, Bell A, and Salih C (2021) Coronary aneurysms, myocardial dysfunction, and shock in a COVID-19 child, role of ECMO, immunomodulation, and cardiac CT. Cardiology in the Young 31: 1043-1047. doi: $10.1017 /$ S1047951121000147

Received: 15 August 2020

Revised: 30 December 2020

Accepted: 4 January 2021

First published online: 28 January 2021

Keywords:

COVID-19; coronary aneurysms; extracorporeal membrane oxygenation; immunomodulation; cardiac CT; multisystem inflammatory syndrome in children

\section{Author for correspondence:}

Muhammad R. Mustafa FRCS(CTh), FETCS, Paediatric Cardiac Surgery, Evelina London Children's Hospital, Guy's and St Thomas' NHS Foundation Trust, Westminster Bridge Road, London SE1 7EH, UK. Tel: +44 2071887188. E-mail: Muhammad.Mustafa@gstt.nhs.uk
Muhammad R. Mustafa ${ }^{1}$ (D), Michael J. Carter ${ }^{2,3}$, James Wong ${ }^{4}$, Aaron Bell ${ }^{4}$ and

Caner Salih ${ }^{1}$

${ }^{1}$ Paediatric Cardiac Surgery Department, Evelina London Children's Hospital, Guy's and St Thomas' NHS Foundation Trust, London, UK; ' ${ }^{2}$ epartment of Women and Children's Health, School of Life Course Sciences, King's College London, London, UK; 'PPaediatric Intensive Care, Evelina London Children's Hospital, Guy's and St Thomas' NHS Foundation Trust, London, UK and ${ }^{4}$ Paediatric Cardiology Department, Evelina London Children's Hospital, Guy's and St Thomas' NHS Foundation Trust, London, UK

\section{Abstract}

Coronary artery aneurysms in children were observed as a rare complication associated with coronavirus disease 2019 (COVID-19). This case report describes the severe end of the spectrum of the new multisystem inflammatory syndrome in a 12 -year-old child with coronary aneurysms, myocardial dysfunction, and shock, managed successfully with extracorporeal membrane oxygenation support and immunomodulation therapy. This report also highlights the additional benefits of cardiac CT in the diagnosis and follow-up of coronary aneurysms.

\section{History of presentation}

In spring 2020 during the coronavirus disease 2019 (COVID-19) pandemic, a 12-year-old girl, weighing $34 \mathrm{~kg}$ and previously fit and well, was transferred to our paediatric ICU from the emergency department in refractory shock. She had presented with persistent fever of $40{ }^{\circ} \mathrm{C}$ for 3 days and abdominal pain, diarrhoea, and vomiting. Her condition failed to respond to $40 \mathrm{~mL} / \mathrm{kg}$ boluses of IV fluids and IV antibiotics. On clinical examination, she had wide pulse pressure, erythematous rashes, lymphadenopathy, conjunctivitis, and cracked lips.

\section{Investigations}

Abdominal CT scan excluded a surgical cause for her deterioration; however, there was free fluid in abdomen and pelvis with peri-portal hepatic oedema and several enlarged mesenteric lymph nodes. Blood tests showed high inflammatory markers, with C-reactive protein $306 \mathrm{mg} / \mathrm{L}$, ferritin $4185 \mathrm{ug} / \mathrm{L}$, D-dimers $69 \mathrm{mg} / \mathrm{L}$, fibrinogen $6 \mathrm{~g} / \mathrm{L}$, procalcitonin $>100 \mathrm{ng} / \mathrm{mL}$, neutrophils $19 \times 10^{9} / \mathrm{L}$, and lymphocytes $0.2 \times 10^{9} / \mathrm{L}$. Cardiac biomarkers were also elevated; troponin $\mathrm{T}$ $43 \mathrm{ng} / \mathrm{L}$ (normal 0-13) and NT-proBNP $14017 \mathrm{ng} / \mathrm{L}$ (normal <400) (Table 1). Blood lactate level was high $(6 \mathrm{mmol} / \mathrm{L})$.

Transthoracic echocardiography immediately following paediatric ICU admission showed a structurally normal heart with severely impaired biventricular systolic function with left ventricle fractional shortening of $10 \%$. Kawasaki disease shock syndrome was considered, and echocardiography showed bright but non-dilated coronary arteries. However, cardiac CT performed on day 4 showed a small fusiform aneurysm of $4.2 \mathrm{~mm}\left(Z\right.$ score $\left.+2.6^{1}\right)$ of the entire length of left main stem and a fusiform aneurysm of $3.5 \mathrm{~mm}(\mathrm{Z}$ score +3.0$)$ of the proximal and middle segments of the left anterior descending (Fig 1). Chest CT showed extensive bilateral patchy consolidation and adjacent ground glass opacification affecting all lobes with a lower lobe predominance. The main pulmonary artery was dilated with no visualised pulmonary embolism (Fig 2).

Cardiac MRI scan was performed on day 14 of hospital admission and showed global increase in T2-weighted imaging indicating myocardial oedema but with normal left ventricle ejection fraction and a normal wall mass.

Severe acute respiratory syndrome coronavirus 2 (SARS-CoV-2) PCR samples from nose, throat, and broncho-alveolar lavage were negative; however, serum IgG was positive. Blood cultures, swabs, and respiratory viral screening were negative. Renal function remained stable throughout the course of the treatment.

\section{Management}

In view of the circulatory instability, the patient was intubated, ventilated, and started on adrenaline and noradrenaline infusions for cardiovascular support. She had an episode 
Table 1. Patient's blood tests on days 1,5 , and 7

\begin{tabular}{|c|c|c|c|}
\hline Blood test & Day 1 & Day 5 & Day 7 \\
\hline CRP & $306 \mathrm{mg} / \mathrm{L}$ & 85 & 53 \\
\hline Ferritin & $4815 \mathrm{ug} / \mathrm{L}$ & 1629 & 1193 \\
\hline D-dimers & $69 \mathrm{mg} / \mathrm{L}$ & 11 & 7.4 \\
\hline Fibrinogen & $6 \mathrm{~g} / \mathrm{L}$ & 4 & 3 \\
\hline Procalcitonin & $>100 \mathrm{ng} / \mathrm{mL}$ & 55 & 10 \\
\hline Neutrophils & $19 \times 10^{9} / \mathrm{L}$ & 13 & 9.7 \\
\hline Lymphocytes & $0.2 \times 10^{9} / \mathrm{L}$ & 1 & 1.1 \\
\hline Troponin T & $43 \mathrm{ng} / \mathrm{L}$ (normal $0-13$ ) & 23 & 29 \\
\hline NT-proBNP & 14017 ng/L (normal <400) & 7214 & 2533 \\
\hline
\end{tabular}

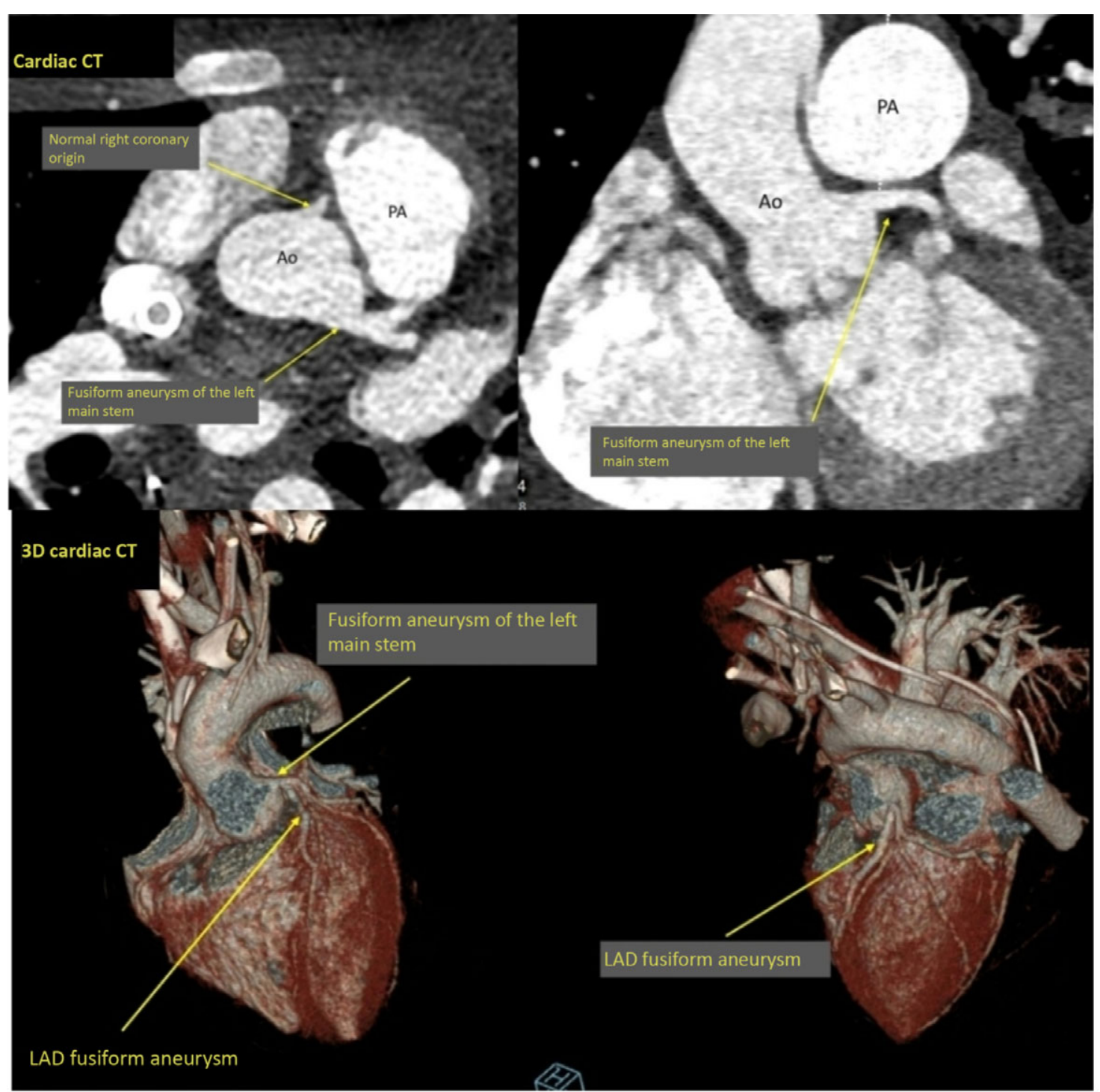

Figure 1. Cardiac CT showing fusiform aneurysm of the left main stem and normal right coronary origin. Ao $=$ aorta, $P A=$ pulmonary artery. $3 D$ Cardiac $C T$ showing fusiform aneurysm of the left main stem, LAD fusiform aneurysm. LAD = left anterior descending.

of sudden bradycardia and hypotension leading to a pulseless electrical activity cardiac arrest 12 hours after admission. Spontaneous circulation returned after 2 minutes of cardiopulmonary resuscitation.
In view of the rapidly worsening cardiovascular instability, and following multidisciplinary team discussion, we decided to put the patient on veno-arterial extracorporeal membrane oxygenation support through cannulation of neck vessel. The right common 

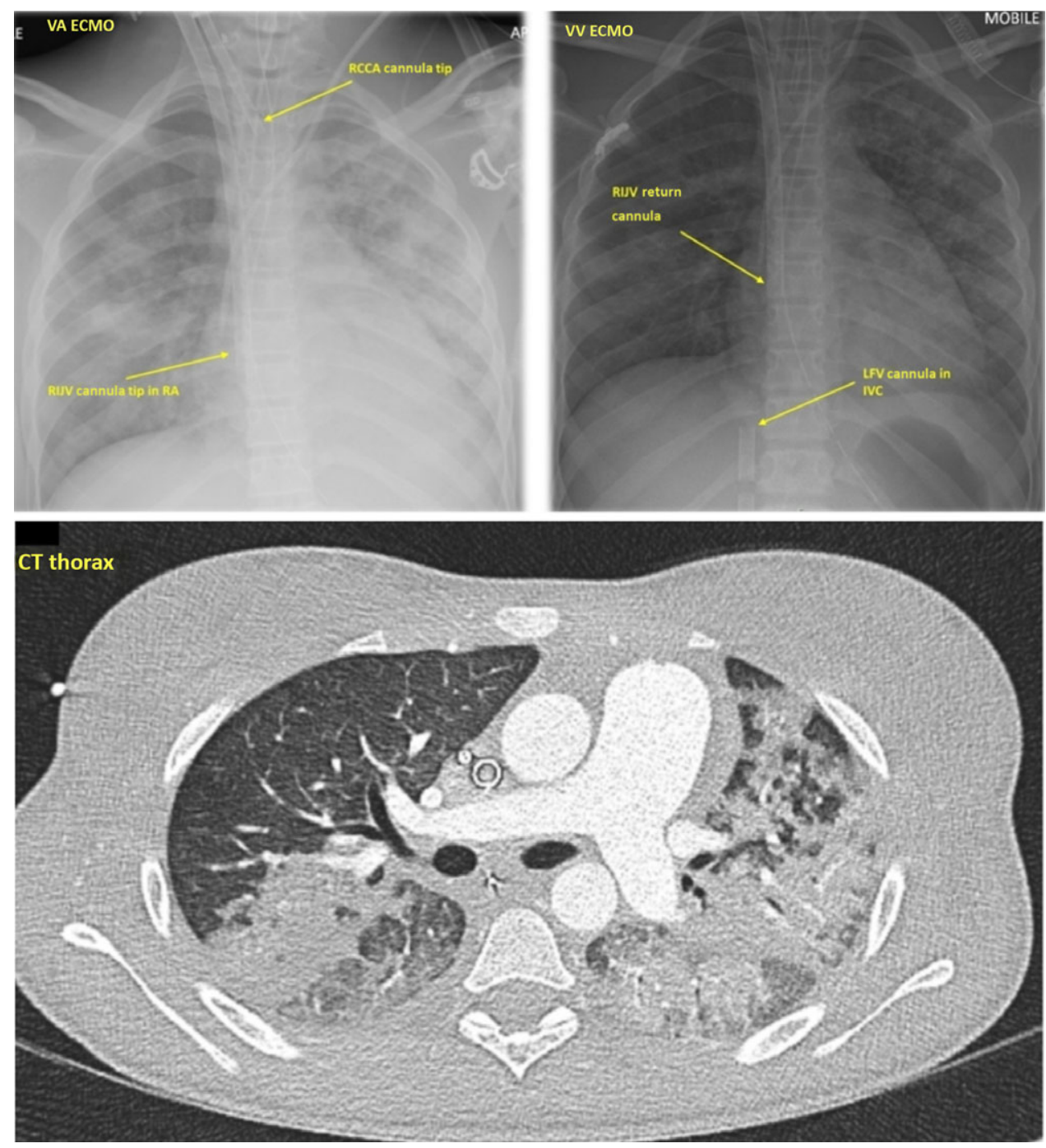

Figure 2. CXR showing VA ECMO with RCCA cannula tip and RIJV cannula tip in RA, W ECMO with RIJV return cannula and LFV cannula in IVC. $V A=$ veno-arterial; $E C M O=$ extracorporeal membrane oxygenation; $\mathrm{RCCA}=$ right common carotid artery; RIJV = right internal jugular vein; $\mathrm{VV}=$ veno-venous; $\mathrm{LFV}=$ left femoral vein; IVC = inferior vena cava.

CT Thorax with extensive bilateral patchy consolidation and adjacent ground glass opacification. carotid artery was cannulated with a 15-Fr Bio-Medicus NextGen femoral arterial cannula (Medtronic Inc.) which was directly inserted with the tip positioned at the proximal brachiocephalic trunk. The right internal jugular vein was subsequently cannulated with a 21-Fr Bio-Medicus NextGen jugular venous cannula (Medtronic Inc.) with its tip positioned in the middle of the right atrium (Fig 2). A flow of $3 \mathrm{~L} /$ minute was achieved and features of shock resolved. Bilateral cerebral near-infrared spectroscopy monitoring was stable at $85-88 \%$. The patient was anticoagulated with heparin infusion at 40 units $/ \mathrm{kg} /$ hour targeting a high anti-Xa level $(0.5-1.0 \mathrm{IU} / \mathrm{mL})$ to prevent thrombosis as d-dimers and fibrinogen were high.

In view of the hyperinflammatory clinical picture, and after multidisciplinary team discussion, the patient was treated with immunomodulation therapy in the form of intravenous immunoglobulin $2 \mathrm{~g} / \mathrm{kg}$ on days 1 and 3, intravenous methylprednisolone $1 \mathrm{~g}$ per day on days $1-5$, aspirin $50 \mathrm{mg} / \mathrm{kg}$ on day 2 , and infliximab (TNF- $\alpha$ antagonist) $6 \mathrm{mg} / \mathrm{kg}$ on day 2 (Table 2 ). Infliximab was chosen for its evidence-based role in refractory Kawasaki disease. ${ }^{2}$ A full course of clindamycin and ceftriaxone antibiotics was also completed.

In combination with levosimendan (inodilator) 0.05-0.1 $\mathrm{mcg} / \mathrm{kg} /$ minute infusion over 24 hours on day 2, cardiac function gradually started to recover, with improved left ventricular ejection and adequate decompression on echocardiography. Despite therapeutic anticoagulation, a thrombus in the right atrium related to the tip of the venous cannula was seen on echocardiogram, which raised the concern of systemic thromboembolism. Following improved cardiac function, but the transient development of hypoxia on trial weaning of veno-arterial extracorporeal membrane oxygenation in conjunction with widespread pulmonary consolidation, the patient was converted to veno-venous extracorporeal membrane oxygenation on day 5. This was achieved following the insertion of a 21-Fr left femoral vein access cannula, with the tip positioned in the inferior vena cava, and the use of the existing right internal jugular vein $21 \mathrm{Fr}$ as a return cannula (Fig 2).

There was continued improvement of all inflammatory markers (Table 1) and veno-venous extracorporeal membrane oxygenation was successfully weaned off on day 7. Mechanical ventilation was weaned, and the patient was extubated the next day. The patient was stepped down from ICU on day 13 and was discharged home 10 days later. She continued oral aspirin, warfarin, and tapering doses of oral prednisolone.

A 6-week follow-up cardiac CT showed normal origin, course, and dimensions of the coronary arteries. The previously described ectasia of the left anterior descending had resolved. Left main was $3.6 \times 3.1 \mathrm{~mm}(Z+1.2)$ and left anterior descending was $2.4 \times 2.2 \mathrm{~mm}(\mathrm{Z}+0.12)$. Lung CT showed a complete resolution 
Table 2. Immunomodulation therapy

\begin{tabular}{lcl}
\hline Medication & Dose & Day \\
\hline Intravenous immunoglobulin (IVIG) & $2 \mathrm{~g} / \mathrm{kg}$ & Days 1 and 3 \\
\hline Intravenous methylprednisolone & $1 \mathrm{~g} \mathrm{per}$ day & Days 1 to 5 \\
\hdashline Aspirin & $50 \mathrm{mg} / \mathrm{kg}$ & Day 2 \\
\hline Infliximab (TNF- $\alpha$ antagonist) & $6 \mathrm{mg} / \mathrm{kg}$ & Day 2 \\
\hline
\end{tabular}

of the previous bilateral multifocal consolidation, while the subtle ground glass changes persisted.

\section{Discussion}

As the whole world is anxiously monitoring the spread of COVID19 and its impact on public health, there is a steep rise in the learning curve of medical teams treating critically ill patients with SARS$\mathrm{CoV}$-2-associated syndromes. More reports are emerging to describe novel presentations in paediatric populations and the role of different therapeutic options for these syndromes.

Although clinical manifestations of infection with SARS-CoV-2 in children are typically less severe than those of adult patients, a small proportion of children develop a hyperinflammatory response with multiorgan involvement - typically several weeks following presumed infection. This condition is termed multisystem inflammatory syndrome in children or paediatric inflammatory multisystem syndrome temporally associated with SARS$\mathrm{CoV}-2 .^{3}$ Multisystem inflammatory syndrome in children may present as a Kawasaki disease syndrome, ${ }^{4}$ with hyperinflammatory shock, cardiogenic shock, and left ventricular dysfunction, ${ }^{5}$ or acute abdomen - making rapid diagnosis problematic. ${ }^{6}$ Coronary artery aneurysms ( $Z$ scores $\geq 2.5$ ) are observed in approximately $10 \%$ of children with multisystem inflammatory syndrome, and acute mortality is $1-2 \%{ }^{7}$ The immunopathologic mechanism of multisystem inflammatory syndrome in children is currently unknown. ${ }^{7}$ This case report describes the severe end of the spectrum of multisystem inflammatory syndrome in children, in a 12-year-old child with coronary aneurysms, myocardial dysfunction, and shock, managed successfully with extracorporeal membrane oxygenation support and immunomodulation therapy. This report also highlights the additional benefits of cardiac CT in the diagnosis and follow-up of coronary artery aneurysms.

Veno-arterial extracorporeal membrane oxygenation support may be a valuable and effective therapeutic option for severe cases of multisystem inflammatory syndrome in children. Our preferred strategy is to establish veno-arterial extracorporeal membrane oxygenation in children through direct cannulation of the right common carotid artery and right internal jugular vein. The neck vessels are readily accessible particularly in urgent clinical situations. Using appropriate size cannulas, full flows can be achieved. We used bilateral near-infrared spectroscopy monitoring to assess for adequate cerebral oxygenation. ${ }^{8}$ Appropriate anticoagulation is of importance as high fibrinogen and d-dimers in this syndrome suggest an increased risk of hypercoagulability, of particular concern in patients receiving extracorporeal membrane oxygenation in whom the risk of thrombotic events is already significant. ${ }^{9,10}$

During the COVID-19 pandemic, a large cohort of children with multisystem inflammatory syndrome in children with cardiac involvement were managed at Evelina London Children's Hospital. Multimodality cardiac imaging with echocardiography, $\mathrm{CT}$, and cardiac MRI was utilised to provide a detailed cardiac assessment. The incidence of coronary involvement was higher on cardiac CT compared with echocardiography. ${ }^{11}$ Cardiac CT may therefore be an important investigation for the identification of patients with coronary artery aneurysms who require close monitoring due to the risk of coronary artery thrombosis and myocardial infarction.

In this case, cardiac CT was performed to delineate coronary anatomy which was not clearly seen on transthoracic echocardiography due to the poor lung acoustic windows from the underlying severe lung disease. An ECG-gated cardiac CT was performed using Siemens SOMATOM Force. And $2 \mathrm{ml} / \mathrm{kg}$ omnipaque was injected at $2 \mathrm{ml} / \mathrm{s}$ and monitoring slices at the mid-descending aorta were used to time maximal contrast opacification of the vessel. A single end-systolic time frame was acquired in expiration with the extracorporeal membrane oxygenation circuit clamped for the duration of the injection.

Coronary artery aneurysms were identified on cardiac CT, despite the initial absence of obvious dilatation on transthoracic echocardiography scans. This emphasises the value of cardiac CT to confirm or rule out coronary artery involvement particularly in extremely sick children with severe myocardial dysfunction and elevated cardiac biomarkers. While echocardiography measures the coronary artery dimension in a single plane, cardiac CT has the advantage of permitting orthogonal imaging of the vessel. ${ }^{12} \mathrm{CT}$ demonstrated that the vessel dimension was found to be enlarged in a tangential dimension to that measured by echocardiography. Furthermore, although initial echocardiographic imaging suggested no aneurysm of the coronary arteries, imaging was limited to only the most proximal coronary segments due to poor acoustic windows and reduced ultrasound penetrance. In contrast, CT is able to show distal coronary artery anatomy with a high degree of spatial resolution. This highlights the use of echocardiography as a screening tool and the additional benefits of performing cardiac CT in those who are most unwell or with the highest suspicion of coronary anomalies. MRI was also able to define the proximal aneurysms but did not have the necessary spatial resolution to define the extent of the aneurysms more distally.

Multisystem inflammatory syndrome in children is a novel immunopathogenic illness, SARS-CoV-2 PCR testing typically (although not always) negative and positive serum IgG in the large majority of cases. The older age group, high prevalence of gastrointestinal symptoms, myocardial dysfunction, and elevated inflammatory markers, cardiac biomarkers, and lymphopenia are distinctive features of this new syndrome. ${ }^{7}$ With the absence of lower respiratory tract symptoms, the chest CT findings are more likely attributable to airway inflammation and/or pulmonary arteritis. The prevalence of respiratory disease is approximately $20 \%$ in multisystem inflammatory syndrome in children. ${ }^{13}$ The precise causal immunological mechanisms for multisystem inflammatory syndrome in children are not yet known. Following the exclusion of other microbial causes, and after multidisciplinary team discussion, the patient was treated with immunomodulation therapy consisting of intravenous immunoglobulin, methylprednisolone, aspirin, and infliximab. The most appropriate treatment strategy is the subject of current research, including the Recovery Trial in the United Kingdom. Nevertheless, immunomodulation therapy has been widely used for multisystem inflammatory syndrome in children with apparent success.

The complete resolution of coronary artery aneurysms after 6 weeks may suggest that the aneurysm formation in multisystem inflammatory syndrome in children is related to fever and 
circulating inflammatory mediators rather than disruption of the arterial wall. ${ }^{3}$

\section{Conclusion}

This severely ill case represents the distinctive clinical and laboratory features of multisystem inflammatory syndrome in children associated with SARS-CoV-2, with the development of coronary artery aneurysms. We highlight the role of extracorporeal membrane oxygenation and immunomodulation therapy in the management of severe multisystem inflammatory syndrome in children and illustrate the additional benefits of performing cardiac CT in those that are most unwell or with the highest suspicion of coronary anomalies. Further research is needed on the mechanism of the disease and the most appropriate treatment strategies.

Acknowledgements. We would like to thank all the doctors and nurses on paediatric ICU, Evelina London Children's Hospital, who participated in the care of this child.

Financial Support. This research received no specific grant from any funding agency, commercial or not-for-profit sectors.

Conflicts of Interest. None.

Ethical Standards. Consent for publication of the case report obtained from the family.

\section{References}

1. Dallaire F, Dahdah N. New equations and a critical appriasal of coronary artery Z scores in healthy children. J Am Soc Echocard 2011; 24: 60-74.
2. Dionne A, Burns JC, Dahdah N, et al. Treatment intensification in patients with Kawasaki disease and coronary artery aneurysms at diagnosis. Pediatrics 2019; 143.

3. Simpson JM, Newburger JW. Multisystem Inflammatory Syndrome in Children in Association With COVID-19. Circulation 2020; 142: 437-440.

4. Verdoni L, Mazza A, Gervasoni A, et al. An outbreak of severe Kawasakilike disease at the Italian epicentre of the SARS-CoV-2 epidemic: an observational cohort study. Lancet 2020; 395: 1771-1778.

5. Riphagen S, Gomez X, Gonzalez-Martinez C, Wilkinson N, Theocharis P. Hyperinflammatory shock in children during COVID-19 pandemic. Lancet 2020; 395: 1607-1608.

6. Belhadjer Z, Méot M, Bajolle F, et al. Acute heart failure in multisystem inflammatory syndrome in children (MIS-C) in the context of global SARS-CoV-2 pandemic. Circulation 2020.

7. Carter MJ, Shankar-Hari M, Tibby SM. Paediatric Inflammatory Multisystem Syndrome Temporally-Associated with SARS-CoV-2 Infection: An Overview. Intensive Care Med 2020.

8. Mustafa MR, Neijenhuis RML, Furci B, Tsang VT. Neck cannulation for bypass in redo sternotomy in children and adults with congenital heart disease. Interact Cardiovasc Thoracis Surg 2020; 31: 108-112, K.

9. Levi M, Thachil J, Iba T, Levy JH. Coagulation abnormalities and thrombosis in patients with COVID-19. Lancet Haematol 2020; 7: e438-440.

10. Johnson K, Jarboe MD, Mychalinska GB, Hirschl RB, Gadepalli SK, Group EE-ENOW. Is there a best approach for extracorporeal life support cannulation: a review of the extracorporeal life support organization. J Pediatr Surg 2018; 53: 1301-1304.

11. Theocharis P, Wong J, Pushparajah K, et al. Multimodality cardiac evaluation in children and young adults with multisystem inflammation associated with COVID-19. Eur Heart J Cardiovasc Imaging 2020.

12. Ghekiere $\mathrm{O}$, Salgado R, Buls N, et al. Image quality in coronary CT angiography: challenges and technical solutions. B J Radiol 2017; 90.

13. Ahmed M, Advani S, Moreira A, et al. Multisystem inflammatory syndrome in children: a systematic review. EClinicalMedicine 2020; 26. 